\title{
Childhood stress impairs social function through AVP-dependent mechanisms
}

\author{
Nichola M. Brydges (10', Jessica Hall², Caroline Best', Lowenna Rule ${ }^{1}$, Holly Watkin', Amanda J. Drake $\mathbb{C}^{3}$, Catrin Lewis², \\ Kerrie L. Thomas ${ }^{1,4}$ and Jeremy Hall,
}

\begin{abstract}
Impaired social function is a core feature of many psychiatric illnesses. Adverse experiences during childhood increase risk for mental illness, however it is currently unclear whether stress early in life plays a direct role in the development of social difficulties. Using a rat model of pre-pubertal stress (PPS), we investigated effects on social behaviour, oxytocin and arginine vasopressin (AVP) in the periphery (plasma) and centrally in the paraventricular and supraoptic hypothalamic nuclei. We also explored social performance and AVP expression (plasma) in participants with borderline personality disorder (BPD) who experienced a high incidence of childhood stress. Social behaviour was impaired and AVP expression increased in animals experiencing PPS and participants with BPD. Behavioural deficits in animals were rescued through administration of the AVPR1a antagonist Relcovaptan (SR49059). AVP levels and recognition of negative emotions were significantly correlated in BPD participants only. In conclusion, early life stress plays a role in the precipitation of social dysfunction, and AVP mediates at least part of this effect.
\end{abstract}

\section{Introduction}

Altered social function is a core component of several adult psychiatric illnesses. For example, depression and schizophrenia are commonly associated with social withdrawal, while major personality disorders can be associated with unstable social relationships (borderline personality disorder, BPD) or aggressive interactions (antisocial personality disorder) ${ }^{1-4}$. Impairments in social behaviour can also be a key determinant of functional outcome in these conditions ${ }^{5-7}$. However, relatively little is known about the causes of impaired social function in psychiatric conditions and their relationship to important aetiological factors associated with psychiatric disorders such as developmental stressors.

Early life stress has been shown to impact on social behaviour and functioning in both animal and human

\footnotetext{
Correspondence: Nichola M. Brydges (brydgesn@cardiff.ac.uk)

${ }^{1}$ Neuroscience and Mental Health Research Institute, Cardiff University, Hadyn Ellis Building, Maindy Road, Cardiff CF24 4HQ, UK

${ }^{2}$ National Centre for Mental Health, Cardiff University, Hadyn Ellis Building, Maindy Road, Cardiff CF24 4HQ, UK

Full list of author information is available at the end of the article.
}

studies $^{8-13}$. In rodent models, social stressors such as social isolation and social defeat during development negatively impact social function in adulthood, and interestingly non-social, physical stressors are also capable of eliciting substantial changes in social function ${ }^{12,14,15}$. The exact nature and timing of stress often influences later outcomes. The majority of studies have focussed on stressors early in development such as maternal separation, but less is known about the effects of stress in the post-weaning, pre-pubertal phase, a time-point suggested as more akin to human childhood ${ }^{16-18}$. During the prepubertal and adolescent phases the limbic system and prefrontal cortex are undergoing significant maturation. These areas are intimately involved in social function and extremely stress reactive due to high densities of corticosteroid receptors ${ }^{19}$. Therefore, stress during this time is predicted to have significant negative impacts on social function. Studies to date have revealed that non-social stress during the peri-pubertal phase results in enhanced aggression, and physical, pre-pubertal stressors alter social interaction in adulthood ${ }^{12,15,20-25}$. In humans, adverse early life experiences have been strongly associated with a 
range of later difficulties in social interaction in longitudinal and cross-sectional studies ${ }^{8,9,26,27}$. In particular, childhood and adolescent stressors have been linked to later social anxiety, withdrawal and aggression, although there is substantial variation in outcomes and not all exposed individuals are affected ${ }^{28-32}$. There is also substantive evidence that childhood adversity is associated with an increased risk for psychiatric disorders in which altered social interaction is a prominent feature, including social anxiety, depression and personality disorders ${ }^{33-35}$. A particularly strong association between developmental adversity and later illness is seen in the case of BPD in which impaired social interactions are a core feature of the presentation ${ }^{34,36-40}$. BPD is also characterised by an unstable sense of self, interpersonal sensitivity, impulsivity, cognitive disturbances (e.g., verbal comprehension, visual attention, working memory and processing speed) and intense, volatile emotions ${ }^{41-43}$. There is currently a lack of valid animal models that translate the key features of BPD, but the role of early life stress has recently been highlighted as a contributing aetiological factor that is amenable to study in animal models ${ }^{44}$. Indeed, certain features of BPD are paralleled by behavioural disturbances following peri-pubertal stress in animal models. For example, rodents exposed to physical or social stress during this period exhibit altered impulsivity and compulsivity and impaired working memory ${ }^{45-47}$.

The mechanisms through which early life stressors impact later social behaviour are still largely unknown. The evolutionarily conserved social neuropeptides oxytocin (OXT) and arginine vasopressin (AVP) are known to play an important role in social behaviour across species $^{48-52}$. AVP and OXT are evolutionarily ancient nonapeptides found in various guises throughout the animal kingdom ${ }^{53}$. In mammals, they are predominantly manufactured in the paraventricular and supraoptic nuclei of the hypothalamus, and released into peripheral circulation via the pituitary gland ${ }^{48}$. OXT and AVP play a wide variety of roles in social behaviour in animals, ranging from inter-male and maternal aggression, conspecific affiliation, social cognition and sexual behaviour ${ }^{48,50,51}$. In humans, intranasal administration of both OXT and AVP influence emotion processing and social cognition, and genetic and functional neuroimaging studies demonstrate a role for OXT and AVP on social behaviour and related limbic brain circuitry ${ }^{54,55}$.

Maternal separation in rodents has been shown to increase AVP levels specifically in the paraventricular nucleus (PVN) and alter OXT levels and OXT/AVP receptor binding in an age and sex specific manner in the offspring ${ }^{17,56-58}$. However, less is known about the effects of stressors applied pre-pubertally, a period which may be more analogous to human childhood, on social peptide regulation. In humans, experience of childhood maltreatment has been associated with decreased levels of OXT in women and men, but the effects of early life stress on AVP levels are less well known ${ }^{59}$.

Here, we report three experiments investigating the relationship between early life stress, AVP/OXT and social behaviour. The aim of experiment 1 was to investigate the effects of pre-pubertal stress (PPS) on social behaviour and levels of OXT and AVP in adult animals. Experiment 2 sought to determine whether changes in social behaviour resulting from PPS could be reversed thorough AVP receptor antagonism, using the AVPR1a antagonist Relcovaptan (SR49059). The third and final experiment assessed peripheral levels of AVP in patients with BPD (a group with a high incidence of childhood adversity) and controls and related these to social behaviour.

\section{Materials and methods \\ Experiments 1 and 2 \\ Subjects}

Female and male Lister Hooded rats were bred in house from adult pairs (Charles River) at Cardiff University. Litters were weaned on postnatal day (PND) 21 and housed in same sex cages $(32 \times 50 \times 21 \mathrm{~cm})$ with littermates. Cages were lined with wood shavings, a cardboard tube and wooden stick were provided as enrichment, light was maintained on a 12:12-h light/dark cycle and food and water were provided ad libitum. All animal experimental methods were carried out in accordance with relevant guidelines and regulations of the European regulations on animal experimentation (Directive 2010/63/ EU) and the UK Home Office Animals (Scientific Procedures) Act 1986. All experimental protocols were approved by the local ethical review body (AWERB) of Cardiff University. Eighty rats (male: 20 control, 20 PPS; female: 20 control, 20 PPS) were used for social testing in experiment 1 , and one hundred and forty four (female: 22 control and vehicle, 24 control and Relcovaptan, 20 PPS and vehicle, 30 PPS and Relcovaptan; male: 12 control and vehicle, 14 control and Relcovaptan, 10 PPS and vehicle, 12 PPS and Relcovaptan) in experiment 2 . In a separate cohort of animals (not subjected to behavioural testing), forty rats (male: 12 control, 10 PPS; female: 8 control, 10 PPS) were used for plasma collection (peripheral AVP), and a further 44 rats (male: 10 control, 13 PPS; female: 10 control, 11 PPS) for immunohistochemistry (central AVP).

\section{Pre-pubertal stress}

PPS was given to half of the litters on PND 25-27. This protocol has been described previously ${ }^{60-62}$, and was originally described by Jacobson-Pick and Richter-Levin ${ }^{63}$. Briefly, animals were given a 10 min swim stress in an opaque swimming tank $(25 \mathrm{~cm}$ high, $34 \mathrm{~cm}$ diameter), 
$12 \mathrm{~L}$ capacity filled with $6 \mathrm{~L}$ of $25 \pm 1{ }^{\circ} \mathrm{C}$ water on PND 25, 3 sessions of $30 \mathrm{~min}$ restraint stress (separated by $30 \mathrm{~min}$ breaks in the home cage) in plastic restraint tubes $(15 \mathrm{~cm}$ length, $5 \mathrm{~cm}$ diameter) on PND 26, and three $30 \mathrm{~min}$ elevated platform exposures (separated by $60 \mathrm{~min}$ breaks in the home cage) on elevated platforms $(15 \times 15 \mathrm{~cm}$, $115 \mathrm{~cm}$ high) on PND 27. Stressors took place in a designated room, separate from the holding room. After PPS, animals were returned to their home cages and holding rooms and left undisturbed (aside from cage cleaning) until early adulthood (PND 60-67). Litters were randomly allocated to experimental groups (PPS or control) based on order of birth. A minimum of five litters per group were used to minimise the effects of pseudoreplication, and litter of origin was accounted for in all statistical analyses.

\section{AVPR1a antagonist}

Relcovaptan (AVPR1a antagonist SR49059, Axon Medchem BV, The Netherlands) was dissolved in 15\% dimethyl sulfoxide (DMSO) and 2\% Tween 80 in $0.9 \%$ saline and administered intraperitoneally at a volume of $2 \mathrm{ml} / \mathrm{kg}$. The dose selected was $1 \mathrm{mg} / \mathrm{kg}$, as this dose reliably inhibits prosocial and autonomic effects of peripherally administered $\mathrm{AVP}^{64,65}$. Vehicle was $15 \%$ DMSO and $2 \%$ Tween 80 in $0.9 \%$ saline.

\section{Social testing}

Testing took place in a clear acrylic tank $(65 \mathrm{~cm} \times$ $65 \mathrm{~cm} \times 40 \mathrm{~cm}$ high) placed on the floor in the centre of a dimly lit room (30 lux). Interactions were filmed from above, and a microphone was suspended above the tank, connected to Avisoft SASLab Pro (avisoft bioacoustics, Germany) to capture ultrasonic vocalisations, which are frequently emitted during murine social encounters. Videos were then watched back by an observer blind to group, and a range of common social behaviours were analysed, as well as latency to initial contact, duration of each contact, number of contacts and total contact time. Specific behaviours recorded were divided into two categories, 'aggressive' consisting of boxing, biting, fighting, mounting, pinning (one rat rolls onto back, and other pins from above), nose off (rats stand immobile facing one another with all four paws on the ground, a defensive strategy), run away, crawl over (one rat crawls over another, often marking with urine), head under body of the other animal, and 'benign or friendly' consisting of allogrooming, sniffing and following. Recorded vocalisations were analysed with Avisoft SASLab Pro programme. Very few $22 \mathrm{kHz}$ vocalisations were produced, so all analyses are based on $50 \mathrm{kHz}$ vocalisations. In rats, $22 \mathrm{kHz}$ vocalisations are most often emitted in aversive situations (e.g., presence of predators, pain), whereas $50 \mathrm{kHz}$ reflect more positive affective states (e.g., during social contact, mating, in response to drugs of abuse or food) ${ }^{66}$.

Three hours before testing, animals were single housed in their holding room, to increase the desire for social contact. One hour before testing they were transferred to the testing room, to habituate them to this environment. In experiment 2 only, half of the animals from each group were given $1 \mathrm{mg} / \mathrm{kg}$ Relcovaptan via intraperitoneal injection $30 \mathrm{~min}$ before testing, the remaining half were administered vehicle only. During testing, two stranger animals were placed into opposite sides of the arena, facing the wall, and allowed to freely interact for $15 \mathrm{~min}$. After this time they were returned to their home cages. The arena was cleaned with ethanol wipes between pairs of animals. Animals were tested in same-sex pairs, with each member of a given pair originating from the same group and treatment condition (PPS or control, vehicle or Relcovaptan) but different litters, so the animals had not previously met. Each pair was treated as one experimental unit for behavioural analysis.

\section{Tissue and plasma collection}

Experiment 1 For plasma AVP and OXT analyses, animals were sacrificed at PND 60 using a rising concentration of $\mathrm{CO}_{2}$, decapitated and trunk blood was collected using EDTA microvette collection tubes (Sarstedt, Germany). Blood was spun at $1500 \times g$ for $10 \mathrm{~min}$, plasma was removed and stored at $-20^{\circ} \mathrm{C}$. For immunohistochemistry, animals were killed at PND 60 by transcardial perfusion with $0.01 \mathrm{M}$ phosphate-buffered saline and 4\% paraformadelhyde (PFA) under anaesthesia for immunohistochemical analysis of AVP in the supraoptic and paraventricular nuclei. Brains were left in PFA overnight $\left(4{ }^{\circ} \mathrm{C}\right)$, then transferred to $30 \%$ sucrose solution for cryoprotection. Coronal $30 \mu \mathrm{m}$ sections were cut through the entire hypothalamic extent on a freezing microtome (Leica RM2245) and placed into a solution of cryoprotectant for storage at $-20^{\circ} \mathrm{C}$ until immunohistochemical analysis.

Experiment 2 After social testing animals were culled (half of the animals were culled $20 \mathrm{~min}$ after testing (direct), the rest 1 week later (delayed)) and trunk blood samples taken as in experiment 1.

\section{ELISA assays}

For experiment 1 , rat plasma samples were assayed untreated. For experiment 2 and all human samples, plasma samples were dried down by adding 2:1 ice-cold acetone:plasma, centrifuging at $3000 \times g$ for $20 \mathrm{~min}$, transferring the supernatant, adding $5 \times$ supernatant volume of ice-cold petroleum ether to the supernatant, centrifuging at $3000 \times g$ for $10 \mathrm{~min}$, discarding the top 
ether layer and drying the remaining aqueous layer under nitrogen gas. This remaining pellet was reconstituted with $250 \mu \mathrm{l}$ of assay buffer and used for ELISA analysis. $\mathrm{Arg}^{8}$-vasopressin and OXT (Enzo Life Sciences, UK) ELISAs were conducted according to the manufacturer's instructions.

\section{Immunohistochemistry}

Three sections per animal per region (supraoptic nucleus (SON) and PVN) were stained for AVP. These were matched for bregma between different animals. Sections were washed between each step for $3 \times 5 \mathrm{~min}$ in $0.01 \mathrm{M}$ Tris-buffered saline (TBS, $\mathrm{pH}$ 7.4) and all steps were carried out at room temperature unless otherwise specified. Sections were blocked with BLOXALL (Vector laboratories, UK) for $10 \mathrm{~min}$, then blocking solution ( $2 \%$ goat serum, $0.3 \%$ Triton- $X$ in $0.01 \mathrm{M}$ TBS) for $60 \mathrm{~min}$, rabbit anti-AVP (1:7500 in blocking solution, AB1565, Millipore, UK) for $24 \mathrm{~h}$ followed by biotinylated goat-antirabbit $(3 \mu \mathrm{g} / \mathrm{ml}$ in blocking solution) for $45 \mathrm{~min}$, then 30 min in VECTASTAIN ABC reagent (avidin-biotinylated horseradish peroxidase complex, Vector Laboratories, UK), before developing in diaminobenzidine solution (Vector Laboratories, UK) for $5 \mathrm{~min}$. Washed sections were mounted onto glass microscope slides and coverslipped with Vectamount (Vector Laboratories, UK). Slides were then imaged at $20 \times$ using an Axio Scan Z1 (Zeiss). The optical density of AVP immunoreactive cells in the supraoptic and paraventricular nuclei were quantified as grey density per area minus background in digitised images using Zen Blue software (Zeiss).

\section{Data analysis}

Data were analysed using generalised linear models in JMP (statistical software, SAS Institute, Cary, NC, USA). Group (control or PPS), sex, Relcovaptan/vehicle (experiment 2 only), direct or delayed sacrifice (experiment 2 only) and all interactions were fitted as factors, and latency to contact, total contact time, average contact duration, specific behaviour (boxing, biting, fighting, mounting, pinning, nose offs, run away, crawl over, allogrooming and following), number and duration of vocalisations, AVP and OXT were fitted as responses. Spearman's Rho was used to assess correlations between behavioural and AVP measures. Unlike AVP measures, it was not possible to obtain individual social data for each animal, as animals were tested in pairs, so the behavioural data for the pair was used in correlational analyses. $P$ was adjusted to 0.004 to account for multiple comparisons.

\section{Experiment 3 Participants}

A total of 20 people with BPD were recruited from the National Centre for Mental Health (NCMH, Welsh
Government funded research centre) participant pool database. Patients with BPD were chosen as this condition is typically associated with very high levels of childhood trauma ${ }^{67}$. A total of 18 healthy age and sex matched controls with no psychiatric disorder were recruited from the community. Exclusion criteria for all participants included current substance dependence, neurological illness, psychotic disorder diagnosis (bipolar I or schizophrenia) and pregnancy, and additionally no psychiatric disorder for control participants. The BPD group consisted of 16 females and 4 males, mean age 43.4 (range: 25-71), controls 14 females and 4 males, mean age 38.4 (range: 20-64). In the BPD group, 11 were being treated with antipsychotic medication and 13 were being treated with antidepressant medication. BPD diagnosis was confirmed in the BPD group and excluded in the controls using the SCID-II (Structured Clinical Interview for DSM Disorders II). Current symptoms of depression were rated using the Hamilton Rating Scale for Depression (HADS). Participants also completed the Childhood Trauma Questionnaire (CTQ), a self-report measure of incidence and severity of childhood trauma consisting of 28 statements relating to one of five subscales of neglect (physical and emotional) or abuse (emotional, physical and sexual). The study was approved by the NHS research ethics committee, and all participants gave informed, written consent, had the opportunity to discuss the study and understood they were free to withdraw at any point.

\section{Social testing}

Participants were given the Ekman 60 faces task, which assess overall emotion recognition performance and identification of basic emotions. During testing, 60 different faces were presented one by one on a computer screen for $3 \mathrm{~s}$, depicting the faces of actors portraying one of six basic emotions (fear, disgust, anger, sadness, happiness and surprise). Ten incidents of each emotion were portrayed during the task. Participants were given as long as needed to identify the emotion. The response choices were shown on the screen, and participants made their selection using a computer mouse. Responses were recorded automatically. Faces were selected from the Ekman and Friesen series of Pictures of Facial Affect ${ }^{68}$, a widely used and validated series of photographs in facial expression research.

\section{Sample collection and analysis}

Participants were visited in their own homes. Blood samples were collected from participants before questionnaire administration and social testing. In total, blood samples were obtained from 17 BPD and 15 controls. Samples were placed in cool bags containing reusable freezer blocks and transferred to the laboratory where they were centrifuged at $16,000 \times g$ for $15 \mathrm{~min}$ at $4{ }^{\circ} \mathrm{C}$. The 
cell-free supernatant plasma fraction was removed and transferred to $-80^{\circ} \mathrm{C}$ storage until analysis. AVP was analysed in plasma following the same protocol as for animal samples, using human $\mathrm{Arg}^{8}$-vasopressin ELISA kit (Enzo Life Sciences, UK). Copeptin was analysed using human copeptin ELISA (Novus Biologicals, UK), according to manufacturer's instructions.

\section{Data analysis}

Data were analysed using generalised linear models or Mann-Whitney $U$ tests in JMP (statistical software, SAS Institute, Cary, NC, USA). Data were checked for normality and homogeneity of variance and transformed to fit these assumptions when necessary. If data could not be transformed, non-parametric tests were used. For AVP and copeptin, group was fitted as a factor with AVP/ copeptin as a response. For the Ekman task, group (control or BPD), emotion (fear, disgust, anger, sadness, happiness and surprise) and group*emotion were fitted as factors, and number of correct responses as the response. Subject was nested within group and fitted as a random factor to account for repeated measures. Due to nonnormal, non-homogenous data, Mann-Whitney $U$ tests were used to analyse responses to the HADS and CTQ questionnaires. Spearman's Rho was used to assess correlations between (i) EKMAN and AVP measures, (ii) CTQ and AVP measures and (iii) CTQ and EKMAN measures. $P$ values were adjusted using the Bonferroni correction to account for multiple comparisons.

\section{Results}

Experiment 1

In experiment 1 we investigated the effects of PPS on social behaviour and AVP expression in adult rats. PPS decreased latency to initial social contact $\left(F_{1,35}=4.27\right.$, $p=0.046)$, decreased duration of each individual contact bout $\left(F_{1,35}=7.68, p=0.009\right)$ and decreased number $\left(F_{1,36}=10.17, p=0.003\right)$ and duration $\left(F_{1,36}=7.78, p=\right.$ $0.008)$ of positively valanced ultrasonic vocalisations $(50 \mathrm{kHz})$, and shortened maximum duration of vocalisations $\left(F_{1,36}=4.13, p=0.049\right)$ in males and females (Fig. 1a-d). No biting or fighting were observed, and only nose-offs, sniffing, crawl overs and follows occurred frequently, with other behaviours rarely observed. There was no effect of PPS on the total number of contacts made $\left(F_{1,35}=2.25, p=0.14\right)$, total contact time $\left(F_{1,35}=1.27, p=0.27\right)$ or time engaged in any specific behaviour (boxing, nose-offs, mounts, pinning, run away crawl over, head under body, allogrooming or following, see Supplementary Table 1 for full statistical results). There were also sex differences in social behaviour, with males displaying increased duration of individual contact bouts $\left(F_{1,35}=7.35, p=0.01\right)$, greater total contact time $\left(F_{1,35}=5.31, p=0.03\right)$, fewer incidents of running away $\left(F_{1,35}=5.53, p=0.02\right)$ and more crawl overs $\left(F_{1,35}=\right.$ $5.23, p=0.03)$, and females generating more ultrasonic vocalisations $\left(F_{1,36}=5.73, p=0.02\right)$ for a longer duration $\left(F_{1,35}=4.73, p=0.04\right)$ and greater maximum duration $\left(F_{1,35}=10.99, p=0.02\right)$.
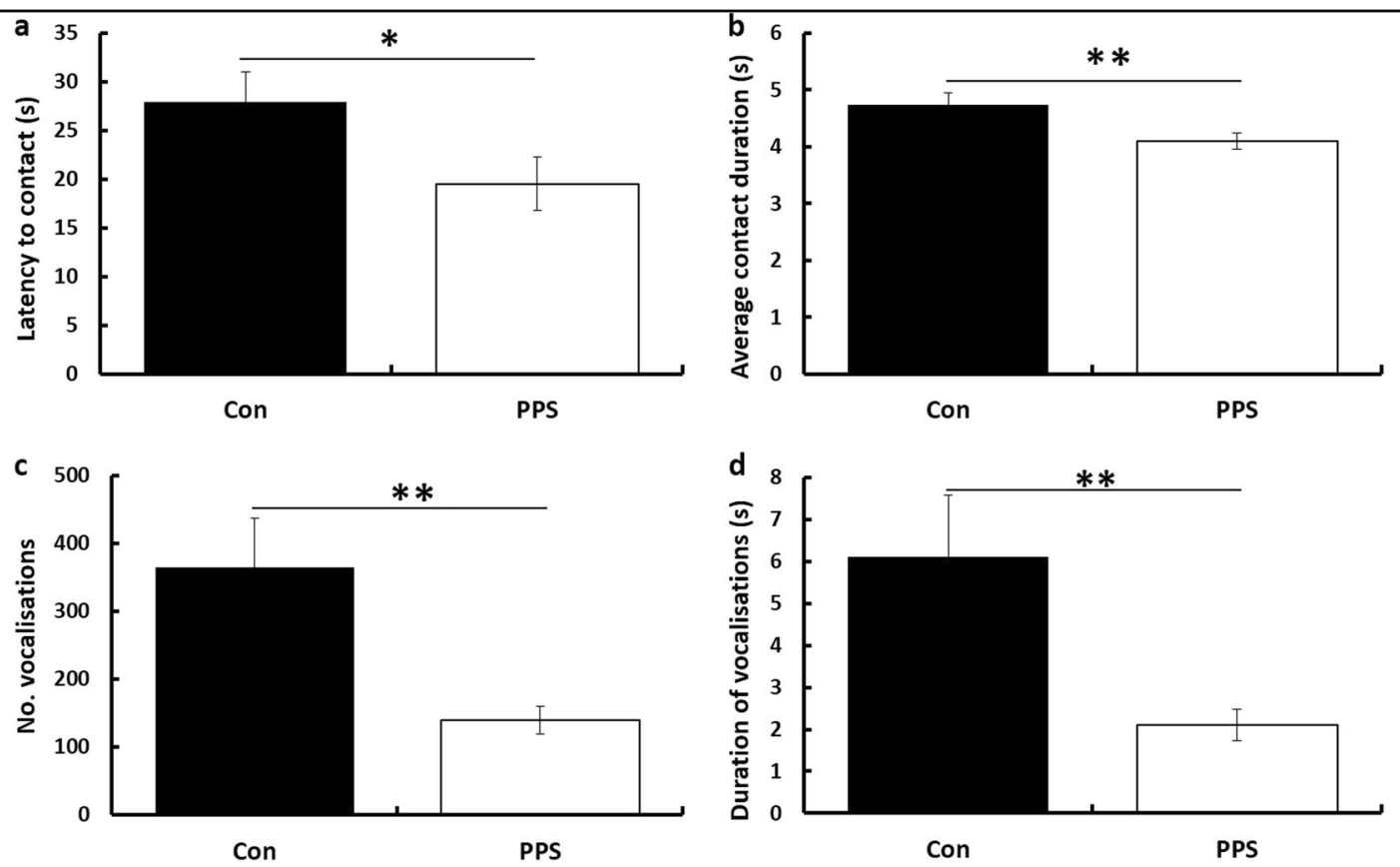

Fig. 1 Social behaviour after PPS. PPS animals were a faster to initiate contact, $\mathbf{b}$ displayed shorter duration of contacts, $\mathbf{c}$ emitted fewer vocalisations which were $\mathbf{d}$ of shorter duration. ${ }^{*} p<0.05,{ }^{* *} p<0.01$. Con control, PPS pre-pubertal stress. Error bars represent 1 S.E. 

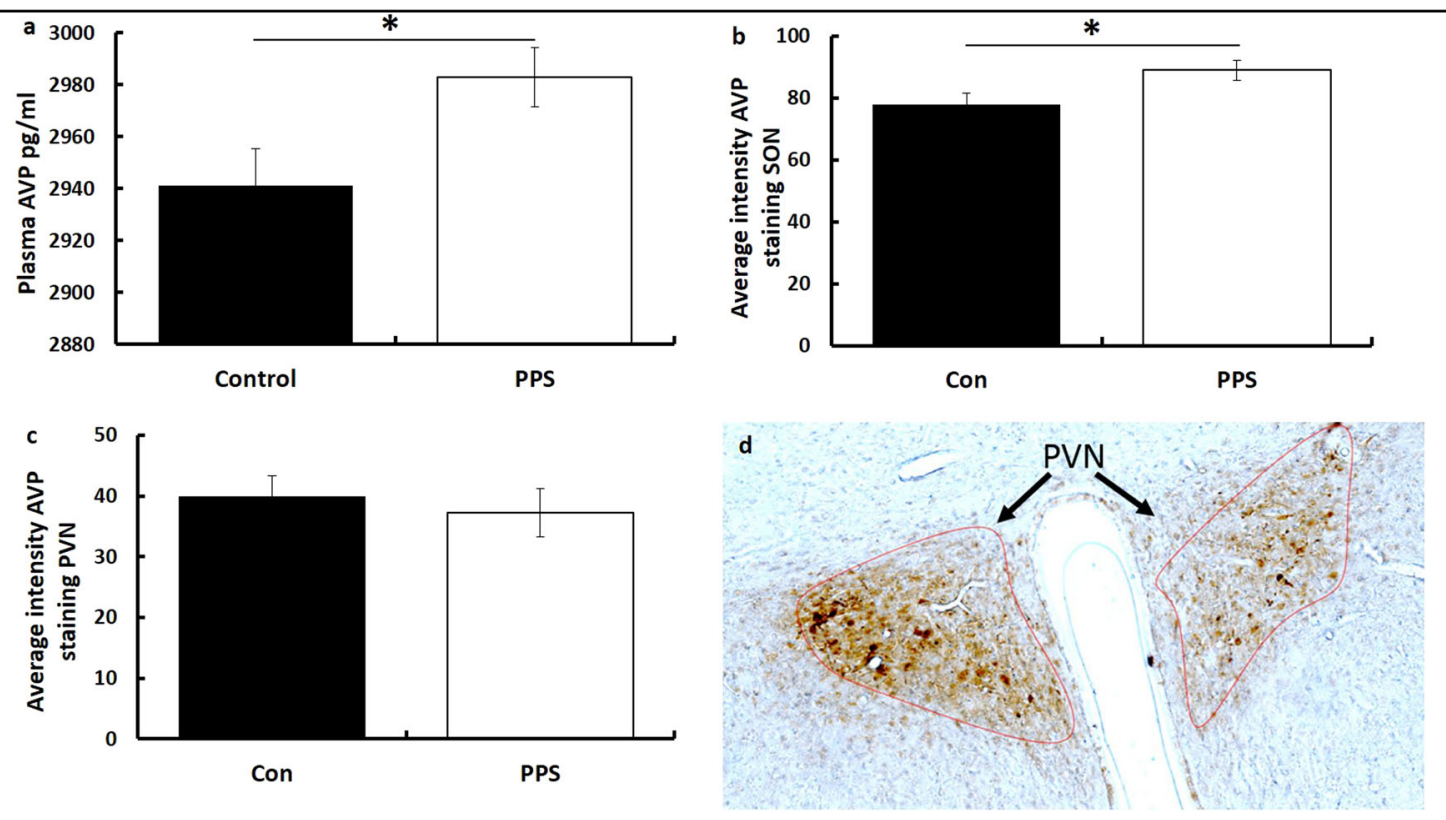

Fig. 2 AVP levels after PPS. Following PPS, levels of AVP were elevated in a plasma and $\mathbf{b}$ supraoptic nucleus but not $\mathbf{c}$ paraventricular nucleus. d A representative immunohistochemistry image. ${ }^{*} p<0.05$. Error bars represent 1 S.E.

In separate cohorts (not behaviourally tested) PPS resulted in higher AVP levels in plasma $\left(F_{1,36}=4.29, p=\right.$ 0.04, Fig. 2a) and centrally in the supraoptic $\left(F_{1,39}=5.31\right.$, $p=0.03$, Fig. 2 b) but not paraventricular $\left(F_{1,36}=0.23\right.$, $P=0.64$, Fig. 2c, d) nucleus of the hypothalamus as assessed by immunohistochemistry. PPS did not influence plasma levels of OXT $\left(F_{1,36}=0.01, p=0.91\right)$. There were no effects of sex on plasma AVP $\left(F_{1,36}=1.42, p=0.24\right)$ or AVP in the supraoptic $\left(F_{1,36}=1.33, p=0.26\right)$ or paraventricular $\left(F_{1,36}=0.09, p=0.76\right)$ nucleus.

\section{Experiment 2}

In experiment 2, we investigated the ability of an AVPR1a antagonist, Relcovaptan, to rescue behavioural changes induced through PPS in our animal model. As in experiment 1, PPS significantly increased plasma levels of AVP in these animals $\left(F_{1,139}=6.95, p=0.01\right.$, Fig. 3a). Replicating our findings in experiment 1 , PPS reduced latency to initial contact and decreased duration of each individual contact during a social test, but administration of Relcovaptan substantially reduced this effect (group"treatment: latency: $F_{1,64}=4.9, p=0.004$, contact: $F_{1,64}=3.1, p=0.03$, data boxcox transformed, Fig. 3b, c). For full statistical results please see Supplementary Table 2a, and for means and standard errors Supplementary Table 2b. There was no correlation between AVP and any behavioural measure in the social task (see Supplementary Table 2a for full statistical results).

\section{Experiment 3}

We next investigated AVP levels and social cognition (assessed using the EKMAN 60 faces task) in participants with BPD, a condition strongly associated with early life stress. There was no significant difference in age or sex between control and BPD groups $\left(F_{1,36}=1.1, p=0.3\right)$. Participants with BPD scored higher on the HADS $(S=$ $192, p<0.001$ ) and CTQ across all abuse domains (emotional abuse: $S=181.5, p<0.0001$; physical abuse: $S=$ 231, $p<0.0001$; sexual abuse: $S=261, p<0.0007$; emotional neglect: $S=196, p<0.0001$; physical neglect: $S=$ $218, p<0.0001)$. Overall, BPD participants were worse at recognising emotion in the EKMAN task $\left(F_{1,36}=7.26\right.$, $p=0.01$, Fig. 4a), replicating some previous findings but contrasting others ${ }^{69-71}$. This was not specific to any emotion, as demonstrated by the lack of group*emotion interaction $\left(F_{5,180}=0.83, p=0.53\right)$. There was also a significant effect of emotion, overall participants were better at recognising happiness, and worse at recognising fear $\left(F_{5,180}=17.23, p<0.0001\right)$. Plasma levels of AVP were higher in BPD compared to control participants $\left(F_{1,28}=5.66, p=0.03\right.$, Fig. $\left.4 \mathrm{~b}\right)$, reflecting the higher peripheral AVP levels seen in the rat PPS model. Measurement of AVP has been suggested as problematic due to rapid clearance from circulation, instability in plasma and high platelet binding ${ }^{72}$. Therefore, we also measured plasma levels of copeptin, the c-terminal segment of the AVP precursor peptide, which has been suggested a more stable, surrogate marker of AVP release ${ }^{73}$. Copeptin was also elevated in the BPD patients $\left(F_{1,28}=4.36, p<0.05\right.$, Fig. $\left.4 \mathrm{c}\right)$. Within the BPD group only there was a negative correlation between plasma AVP and number of errors in recognising threat-related emotions, such that higher AVP corresponded with enhanced responsiveness to negative emotions (average 

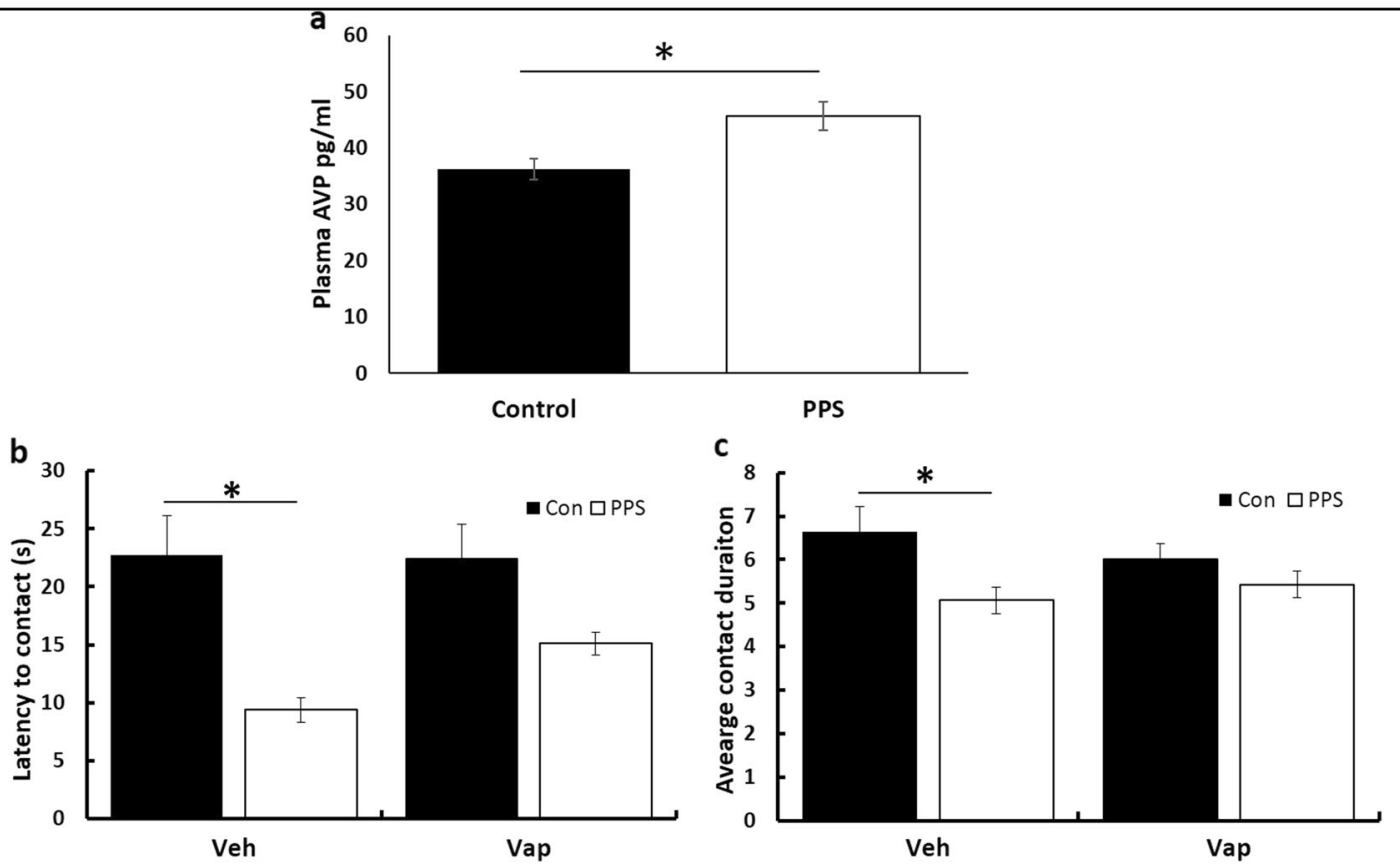

Fig. 3 Effects of Relcovaptan on social behaviour after PPS. a Following PPS plasma levels of AVP were again elevated. PPS animals were $\mathbf{b}$ faster to initiate contact, this was reversed by administration of Relcovaptan, and $\mathbf{c}$ displayed shorter duration of contacts, which was again reversed by Relcovaptan. ${ }^{*} p<0.05$. Error bars represent 1 S.E.
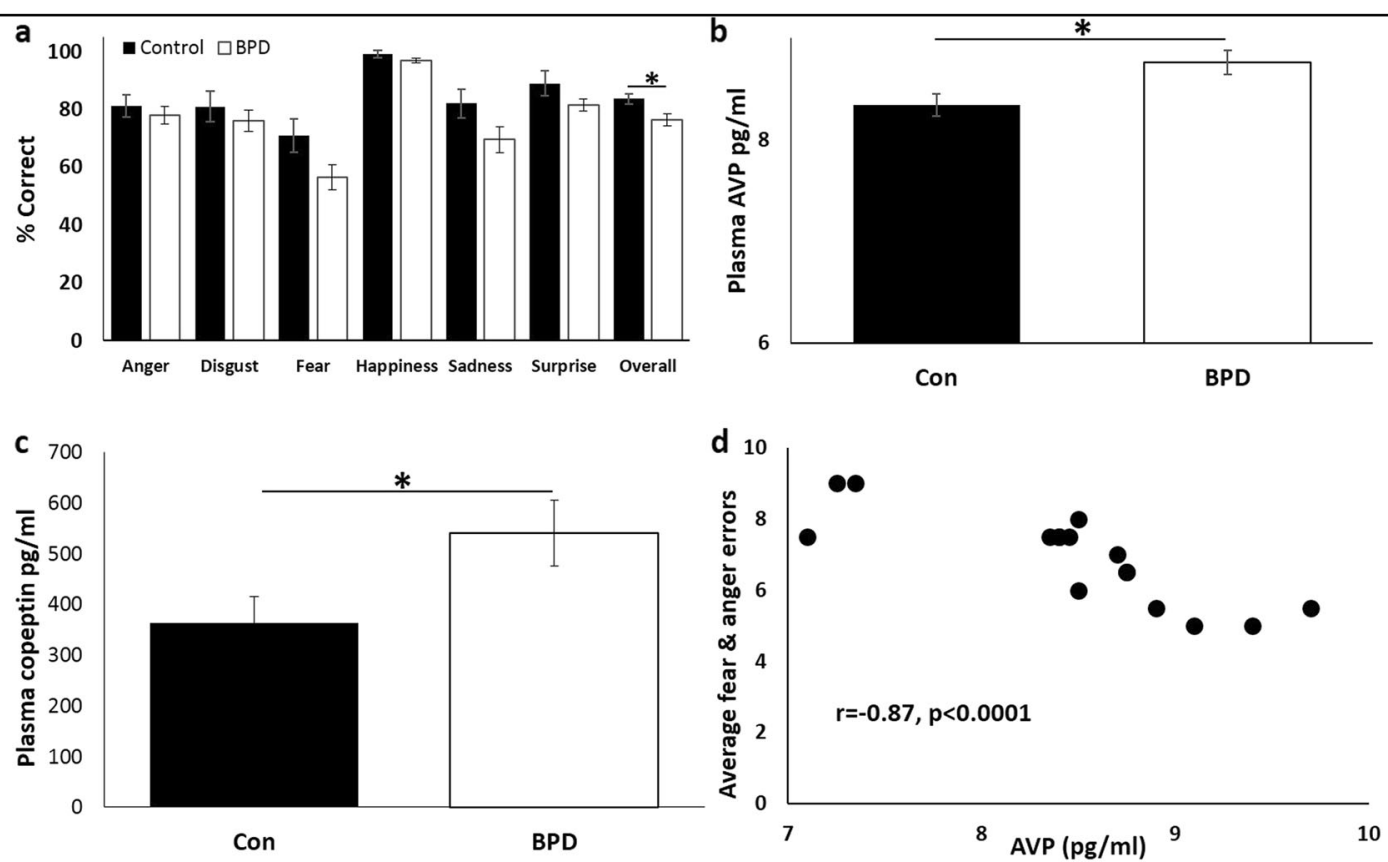

Fig. 4 Social behaviour and AVP levels in BPD and controls. BPD participants were a worse at recognising emotion in the EKMAN task, $\mathbf{b}$ had higher plasma levels of AVP and $\mathbf{c}$ copeptin, and $\mathbf{d}$ within the BPD group only, higher AVP correlated with enhanced detection of negative emotions (fear and anger). * $p<0.05$. Error bars represent 1 S.E.

of fear and anger, $r_{\mathrm{S}}=-0.87, p<0.0001$, Fig. 4d). Higher levels of emotional neglect were correlated with an increased ability to recognise anger $\left(r_{\mathrm{S}}=0.7, p<0.001\right)$ within the
BPD group. There were no other correlations between AVP, emotion recognition and scores on the CTQ (see Supplementary Table 3 for full statistical report). 


\section{Discussion}

PPS resulted in altered social behaviour and elevated protein levels of AVP peripherally in plasma and centrally in the SON in rats. Relcovaptan, an AVPR1a antagonist, reversed behavioural changes induced by PPS. Similarly, individuals with BPD, a population with a high incidence of childhood adversity, had elevated plasma AVP and altered performance on tests of social emotion recognition. Furthermore, AVP levels correlated with increased sensitivity to negative emotional expressions in the BPD group. This suggests the AVP system is at least one mechanism through which early life stress can result in social difficulties in adulthood.

In our animal model, PPS resulted in a sustained increase in AVP levels both peripherally (blood plasma) and centrally (SON). AVP is mainly synthesised in the magnocellular neurons of the SON and PVN and parvocellular neurons of the $\mathrm{PVN}^{74}$. Parvocellular neurons project to the zona externa of the median eminence, and AVP originating from these neurons is suggested to be intimately involved in neuro-endocrine HPA axis function at rest and following acute and chronic stress ${ }^{75,76}$. In contrast, magnocellular neurons project through the zona interna of the median eminence to the posterior pituitary, where AVP is stored in axon terminals and upon stimulation, secreted into systemic blood circulation. This suggests that increased peripheral AVP in our PPS animals is a direct result of the observed upregulation in the magnocellular neurons of the SON. Previously, early life stress in the form of maternal separation increased AVP expression in the PVN of adult mice, but levels in the SON were unaffected ${ }^{57}$. It is unknown whether elevated PVN AVP would produce similar peripheral increases following early life stress as the previous maternal separation study did not examine peripheral AVP levels.

The differential effect of maternal separation and later juvenile stress on AVP in different nuclei of the hypothalamus could result from differences in the stress protocol (short-term physical stress vs. maternal separation) or the developmental time point (PND 25-27 vs. PND $1-10)$. This highlights the importance of timing-it is likely that different structures and processes are vulnerable to stressful perturbations at different time points during development. The nature of the stress is also likely to produce different effects in SON and PVN-in adult rats exposure to cold and heat stress increases AVP expression in the PVN, whereas SON AVP only responds to cold stress ${ }^{77}$. Furthermore, differences in the AVP system following early life stress may not be apparent until the system is challenged in a specific manner. AVP was increased in the SON and PVN of maternally separated adult rats and the SON of female prairie voles subjected to chronic social isolation only after experience of stressful intrasexual social, but not non-social, tests ${ }^{78-80}$.
In contrast to AVP, we found no changes in OXT following PPS. OXT is also produced in the SON and PVN and responds to certain early life stressors. In rodents, prenatal stress reduces OXT in the PVN, whereas maternal separation has no effect (although OXT receptor binding is altered $)^{13,56,81-83}$. In humans, lower levels of OXT have been detected after severe childhood maltreatment in women, and after early life stress before the age of 12 but not during adolescence in $\operatorname{men}^{84,85}$. Conversely, less severe forms of childhood physical abuse result in increased OXT in adulthood ${ }^{86}$, again highlighting the likely importance of stressor nature and timing for later outcomes.

During a social interaction test, rats exposed to PPS displayed decreased latency to initial contact and decreased duration of individual contact bouts, and number and duration of positively valanced ultrasonic vocalisations were also reduced. Previous work has found that PPS decreases conspecific social interaction in adult males interacting with both adults (rats) ${ }^{21,23}$ and juveniles (mice) ${ }^{20}$ (but see ref. ${ }^{87}$ ). In animals, AVP is important for social behaviour: disruption of AVP neurons impairs social memory specifically, and $\mathrm{AVPR}^{-1-} \mathrm{a}^{-1}$ and $\mathrm{AVPR}_{1} \mathrm{~b}^{-1-}$ mice demonstrate aberrant social recognition ${ }^{88-90}$. AVP infusion into the olfactory bulb and lateral septum facilitates social recognition memory in rats, and along with AVPR1a, AVP modulates aggression through several brain regions ${ }^{51,91-94}$. This raised the question of whether increased AVP following PPS in our animal model was directly responsible for altered social behaviour. We therefore investigated the ability of an AVPR1a antagonist, Relcovaptan (SR49059), to rescue behaviour following PPS. Social behaviour resulting from PPS was partially restored by administration of Relcovaptan, suggesting that AVP mediates some of the effects of PPS on adulthood social behaviour, and that this is a reversible phenomenon. Other neurobiological systems and receptors may also mediate the effects of PPS on later social behaviour. AVP can also exert effects through AVP receptor $1 \mathrm{~b}$ and the OXT receptor to influence social behaviour, and future experiments should address the contribution of these receptor subtypes to the social phenotype observed following PPS. The involvement of other hormones with a known role in social behaviour, particularly those which are known to be responsive to early life stress (e.g., monoamines, corticotrophin releasing hormone and glucocorticoids) ${ }^{12}$ should similarly be investigated.

We next investigated whether AVP levels are also elevated in a psychiatric condition associated with high levels of childhood adversity, namely BPD. As with our animal model, we find that AVP protein was elevated in the plasma of BPD participants. Concordant with animal research, AVP and AVPR1a are strongly implicated in 
human social behaviour. AVPR1a promoter polymorphisms (particularly in RS3 microsatellite repeats) are associated with variation in prosocial behaviour, especially empathy and altruism, and polymorphisms in AVPR1a and AVPR1b have been shown to associate with social dysfunction in autism spectrum disorder ${ }^{83,95-98}$. In the present study, BPD participants demonstrated altered social behaviour. Specifically, they displayed decreased sensitivity to recognising facial expressions of emotion in the Ekman task when compared to controls. This supports previous findings of impaired social cognition in $\mathrm{BPD}^{10,36,40,69,71}$. We also find that higher AVP is correlated with higher sensitivity to detect fear and anger in this group, suggesting a role for AVP in the recognition of emotions directly related to threat perception in BPD. Interestingly, BPD are a group already known to display heightened attention to threat related social cues ${ }^{41}$. In agreement with our findings, in healthy individuals, some studies have found that intranasal administration of AVP enhances threat perception and detection of anger ${ }^{99,100}$. Others have found the opposite effect, with AVP administration decreasing recognition of negative emotions in men $^{101}$. The precise effects may be affected by the particular group under study: intranasal AVP decreases anger detection in schizophrenic men, yet improves fear recognition in schizophrenic women ${ }^{101}$. Based on our current results it may be predicted that AVPR1a antagonism would further impair recognition of fear and anger in the BPD group, but we cannot predict the consequences for recognising other emotions. This would be an interesting area for future research.

We found no sex differences in response to PPS across any of our measures, with males and females being equally affected. There are well-known sex-differences in the AVP and OXT systems, for example the expression of AVP and AVPR1a are typically higher in males, and the administration of AVP and AVPR1a antagonists can produce differing effects in males and females ${ }^{102}$. Previous research in our laboratory has demonstrated striking sex differences in response to our PPS paradigm, for example males display impaired fear conditioning and altered hippocampal neurogenesis, whereas females are unaffected ${ }^{103}$. Conversely, PPS females show greater alterations in the hypothalamic-pituitary-adrenal axis ${ }^{104}$. This demonstrates that males and females display sex-based vulnerabilities and resiliencies to the lasting effects of PPS in certain domains, but are equally affected in others, highlighting the need to consider males and females in pre-clinical and clinical studies. In the social interaction task we found sex differences in behaviour regardless of treatment, with males spending more time in contact (increased average duration of contact and total contact time) and females vocalising more and performing more crawl overs and run-aways (see Supplementary Table 1).
This translational study provides evidence from across species for the importance of AVP in relation to early life stress and social behaviour. Of particular interest is the fact that despite the different stressor types administered in the animal model and experienced by the BPD cohort, both populations experienced increased AVP and altered social behaviour. The exact contribution and consequences of different stressor types to the precipitation of behavioural and biological alterations later in life are not fully understood, and should form the basis of future research. Our results demonstrate that the AVP system may be a useful target for ameliorating social difficulties in conditions associated with high rates of childhood trauma, such as BPD, and that peripheral AVP levels may represent a valuable biomarker for stratification of treatment in such groups. As a high rate of childhood adversity is found in other psychiatric populations, for example depression and anxiety, these would be valuable cohorts for future study. Future studies should also aim to measure broader domains of social function, to further align social measures between human and animal studies. Additional scales and self-reports of social interaction would be informative in human populations, and the response of PPS animals to aversive social situations such as tests of social dominance and resident-intruder paradigms would prove informative and enhance cross-species translation.

In conclusion, we find that experience of stressful events early in life is likely to play a role in the development of social abnormalities in adulthood, and alterations in the AVP system are at least partially responsible for this effect. AVPR1a is a promising target for intervention in disorders with a social phenotype, especially those produced thorough stressful early life experiences.

\section{Acknowledgements}

We wish to acknowledge support from the Cardiff University Neuroscience and Mental Health Research Institute and The Jane Hodge Foundation who provided NB with fellowship funding during this research, as well as The Waterloo Foundation who provided grant funding for preliminary work (Grant number 918-1875). We would also like to thank Dr. Oscar Almer for constructing the social testing arena, and Dr. Stephane Baudouin for loan of audio recording and analysis equipment. We thank the National Centre for Mental Health $(\mathrm{NCMH})$, which is a collaboration between Cardiff, Swansea and Bangor Universities and is funded by Welsh Government through Health and Care Research Wales. We thank NCMH study participants for their invaluable contribution to this project. Remaining authors declare no competing interests.

\section{Author details}

'Neuroscience and Mental Health Research Institute, Cardiff University, Hadyn Ellis Building, Maindy Road, Cardiff CF24 4HQ, UK. ${ }^{2}$ National Centre for Mental Health, Cardiff University, Hadyn Ellis Building, Maindy Road, Cardiff CF24 4HQ, UK. ${ }^{3}$ University/BHF Centre for Cardiovascular Science, University of Edinburgh, Edinburgh, UK. ${ }^{4}$ School of Biosciences, Cardiff University, Museum Avenue, Cardiff CF10 3AX, UK. ${ }^{5}$ MRC Centre for Neuropsychiatric Genetics and Genomics, Cardiff University, Hadyn Ellis Building, Maindy Road, Cardiff CF24 $4 \mathrm{HQ}, \mathrm{UK}$ 


\section{Author contributions}

N.B. and Jess H. contributed to study design, experimental work, data analysis and paper writing. C.B. contributed to experimental work and paper writing, L.R. and H.W. contributed to the experimental work, data analysis and paper writing, and Jeremy H. contributed to study design and paper writing, A.D. and K.L.T. contributed to paper writing.

\section{Competing interests}

The authors declare no competing interests.

\section{Publisher's note}

Springer Nature remains neutral with regard to jurisdictional claims in published maps and institutional affiliations.

Supplementary Information accompanies this paper at (https://doi.org/ 10.1038/s41398-019-0678-0).

Received: 21 August 2019 Revised: 19 November 2019 Accepted: 27 November 2019

Published online: 09 December 2019

\section{References}

1. Green, M. F., Horan, W. P. \& Lee, J. Social cognition in schizophrenia. Nat. Rev. Neurosci. 16, 620-631 (2015).

2. Lieb, K., Zanarini, M. C., Schmahl, C., Linehan, M. M. \& Bohus, M. Borderline personality disorder. Lancet 364, 453-461 (2004).

3. Bora, E. \& Pantelis, C. Social cognition in schizophrenia in comparison to bipolar disorder: a meta-analysis. Schizophrenia Res. 175, 72-78 (2016).

4. Raine, A. Antisocial personality as a neurodevelopmental disorder. Annu. Rev. Clin. Psychol. 14, 259-289 (2018).

5. Green, M. F., Llerena, K. \& Kern, R. S. The "Right Stuff" revisited: what have we learned about the determinants of daily functioning in schizophrenia? Schizophrenia Bull. 41, 781-785 (2015).

6. Javed, A. \& Charles, A. The importance of social cognition in improving functional outcomes in schizophrenia. Front. Psychiatry 9, 14 (2018).

7. Vlad, M., Raucher-Chene, D., Henry, A. \& Kaladjian, A. Functional outcome and social cognition in bipolar disorder: Is there a connection? Eur. Psychiatry 52, 116-125 (2018).

8. Stain, H. J. et al. Impact of interpersonal trauma on the social functioning of adults with first-episode psychosis. Schizophrenia Bull. 40, 1491-1498 (2014).

9. Palmier-Claus, J. et al. Childhood adversity and social functioning in psychosis: exploring clinical and cognitive mediators. Psychiatry Res. 238, 25-32 (2016).

10. Nicol, K., Pope, M., Sprengelmeyer, R., Young, A. W. \& Hall, J. Social judgement in borderline personality disorder. Plos ONE 8, e73440 (2013).

11. Nicol, K., Pope, M., Romaniuk, L. \& Hall, J. Childhood trauma, midbrain activation and psychotic symptoms in borderline personality disorder. Transl. Psychiatry 5, e559 (2015).

12. Sandi, C. \& Haller, J. Stress and the social brain: behavioural effects and neurobiological mechanisms. Nat. Rev. Neurosci. 16, 290-304 (2015).

13. de Souza, M. A. et al. Prenatal stress produces social behavior deficits and alters the number of oxytocin and vasopressin neurons in adult rats. Neurochem. Res. 38, 1479-1489 (2013).

14. Zhang, F., Yuan, S. N., Shao, F. \& Wang, W. W. Adolescent social defeat induced alterations in social behavior and cognitive flexibility in adult mice: effects of developmental stage and social condition. Front. Behav. Neurosci. 10, 149 (2016).

15. Toth, M., Halasz, J., Mikics, E., Barsy, B. \& Haller, J. Early social deprivation induces disturdbed social communication and violent aggression in adulthood. Behav. Neurosci. 122, 849-854 (2008).

16. Brydges, N. M. Pre-pubertal stress and brain development in rodents. Curr. Opin. Behav. Sci. 7, 8-14 (2016)

17. Veenema, A. H. \& Neumann, I. D. Maternal separation enhances offensive play-fighting, basal corticosterone and hypothalamic vasopressin mRNA expression in juvenile mate rats. Psychoneuroendocrinology 34, 463-467 (2009).
18. Kentrop, J. et al. Effects of maternal deprivation and complex housing on rat social behavior in adolescence and adulthood. Front. Behav. Neurosci. 12, 193 (2018).

19. Herman, J. P. Regulation of adrenocorticosteroid receptor messenger-RNA expression in the central nervous system. Cell. Mol. Neurobiol. 13, 349-372 (1993).

20. Jacobson-Pick, S., Audet, M. C., Nathoo, N. \& Anisman, H. Stressor experiences during the juvenile period increase stressor responsivity in adulthood: transmission of stressor experiences. Behavioural Brain Res. 216, 365-374 (2011).

21. Toth, E., Avital, A., Leshem, M., Richter-Levin, G. \& Braun, K. Neonatal and juvenile stress induces changes in adult social behavior without affecting cognitive function. Behavioural Brain Res. 190, 135-139 (2008).

22. Ueno, $H$. et al. Juvenile stress induces behavioral change and affects perineuronal net formation in juvenile mice. Bmc Neurosci. 19, 41 (2018).

23. Mackay, J. C. et al. Protracted effects of juvenile stressor exposure are mitigated by access to palatable food. Plos ONE 9, e96573 (2014).

24. Marquez, C. et al. Peripuberty stress leads to abnormal aggression, altered amygdala and orbitofrontal reactivity and increased prefrontal MAOA gene expression. Transl. Psychiatry 3, e216 (2013).

25. Cordero, M. I., Ansermet, F. \& Sandi, C. Long-term programming of enhanced aggression by peripuberty stress in female rats. Psychoneuroendocrinology $\mathbf{3 8}$, 2758-2769 (2013).

26. Kilian, S. et al. Childhood adversity and cognitive function in schizophrenia spectrum disorders and healthy controls: evidence for an association between neglect and social cognition. Psychol. Med. 48, 2186-2193 (2018).

27. Chu, D. A., Bryant, R. A., Gatt, J. M. \& Harris, A. W. F. Failure to differentiate between threat-related and positive emotion cues in healthy adults with childhood interpersonal or adult trauma. J. Psychiatr. Res. 78, 31-41 (2016).

28. Fernandes, V. \& Osorio, F. L. Are there associations between early emotional trauma and anxiety disorders? Evidence from a systematic literature review and meta-analysis. Eur. Psychiatry 30, 756-764 (2015).

29. Haller, J., Harold, G., Sandi, C. \& Neumann, I. D. Effects of adverse early-life events on aggression and anti-social behaviours in animals and humans. $J$. Neuroendocrinol. 26, 724-738 (2014).

30. McGrath, J. J. et al. The association between childhood adversities and subsequent first onset of psychotic experiences: a cross-national analysis of 23998 respondents from 17 countries. Psychol. Med. 47, 1230-1245 (2017).

31. Kessler, R. C. et al. Childhood adversities and adult psychopathology in the WHO World Mental Health Surveys. Br. J. Psychiatry 197, 378-385 (2010).

32. Beutel, M. E. et al. Childhood adversities and distress - the role of resilience in a representative sample. Plos ONE 12, e0173826 (2017).

33. Johnson, J. G., Cohen, P., Brown, J., Smailes, E. M. \& Bernstein, D. P. Childhood maltreatment increases risk for personality disorders during early adulthood. Arch. Gen. Psychiatry 56, 600-606 (1999).

34. Ball, J. S. \& Links, P. S. Borderline personality disorder and childhood trauma: evidence for a causal relationship. Curr. Psychiatry Rep. 11, 63-68 (2009).

35. Bailey, T. et al. Childhood trauma is associated with severity of hallucinations and delusions in psychotic disorders: a systematic review and meta-analysis. Schizophrenia Bull. 44, 1111-1122 (2018).

36. Preissler, S., Dziobek, l., Ritter, K., Heekeren, H. R. \& Roepke, S. Social cognition in borderline personality disorder: evidence for disturbed recognition of the emotions, thoughts, and intentions of others. Front. Behav. Neurosci. 4, 182 (2010).

37. Paris, J. The etiology of borderline personality disorder-a biopsychosocial approach. Psychiatry 57, 316-325 (1994).

38. Battle, C. L. et al. Childhood maltreatment associated with adult personality disorders: findings from the collaborative longitudinal personality disorders study. J. Personal. Disord. 18, 193-211 (2004).

39. Wilson, S., Stroud, C. B. \& Durbin, C. E. Interpersonal dysfunction in personality disorders: a meta-analytic review. Psychological Bull. 143, 677-734 (2017).

40. Lazarus, S. A., Cheavens, J. S., Festa, F. \& Rosenthal, M. Z. Interpersonal functioning in borderline personality disorder: a systematic review of behavioral and laboratory-based assessments. Clin. Psychol. Rev. 34, 193-205 (2014).

41. Gunderson, J. G., Herpertz, S. C., Skodol, A. E., Torgersen, S. \& Zanarini, M. C. Borderline personality disorder. Nat. Rev. Dis. Prim. 4, 18030 (2018).

42. Bazanis, E. et al. Neurocognitive deficits in decision-making and planning of patients with DSM-III-R borderline personality disorder. Psychol. Med. 32, 1395-1405 (2002). 
43. Thomsen, M. S., Ruocco, A. C., Carcone, D., Mathiesen, B. B. \& Simonsen, E. Neurocognitive deficits in borderline personality disorder: associations with childhood trauma and dimensions of personality psychopathology. J. Personal. Disord. 31, 503-521 (2017).

44. Corniquel, M. B., Koenigsberg, H. W. \& Likhtik, E. Toward an animal model of borderline personality disorder. Psychopharmacology 236, 2485-2500 (2019).

45. Brydges, N. M., Holmes, M. C., Harris, A. P., Cardinal, R. N. \& Hall, J. Early life stress produces compulsive-like, but not impulsive, behavior in females. Behav. Neurosci. 129, 300-308 (2015).

46. Gonzalez-Martinez, L. F., D'Aigle, J., Lee, S. M., Lee, H. J. \& Delville, Y. Social stress in early puberty has long-term impacts on impulsive action. Behav. Neurosci. 131, 249-261 (2017).

47. Isgor, C., Kabbaj, M., Akil, H. \& Watson, S. J. Delayed effects of chronic variable stress during peripubertal-juvenile period on hippocampal morphology and on cognitive and stress axis functions in rats. Hippocampus 14, 636-648 (2004)

48. Albers, H. E. The regulation of social recognition, social communication and aggression: vasopressin in the social behavior neural network. Hormones Behav. 61, 283-292 (2012).

49. Albers, H. E. Species, sex and individual differences in the vasotocin/vasopressin system: relationship to neurochemical signaling in the social behavior neural network. Front. Neuroendocrinol. 36, 49-71 (2015).

50. Caldwell, H. K. \& Albers, H. E. Oxytocin, vasopressin, and the motivational forces that drive social behaviors. Curr. Top. Behav. Neurosci. 27, 51-103 (2016).

51. Caldwell, H. K. Oxytocin and vasopressin: powerful regulators of social behavior. Neuroscientist 23, 517-528 (2017).

52. Insel, T. R. The challenge of translation in social neuroscience: a review of oxytocin, vasopressin, and affiliative behavior. Neuron 65, 768-779 (2010).

53. Donaldson, Z. R. \& Young, L. J. Oxytocin, vasopressin, and the neurogenetics of sociality. Science 323, 1429-1429 (2009).

54. Grace, S. A., Rossell, S. L., Heinrichs, M., Kordsachia, C. \& Labuschagne, I. Oxytocin and brain activity in humans: a systematic review and coordinatebased meta-analysis of functional MRI studies. Psychoneuroendocrinology 96, 6-24 (2018).

55. Lefevre, A., Hurlemann, R. \& Grinevich, V. Imaging neuropeptide effects on human brain function. Cell Tissue Res. 375, 279-286 (2018).

56. Lukas, M., Bredewold, R., Neumann, I. D. \& Veenema, A. H. Maternal separation interferes with developmental changes in brain vasopressin and oxytocin receptor binding in male rats. Neuropharmacology 58, 78-87 (2010).

57. Murgatroyd, C. et al. Dynamic DNA methylation programs persistent adverse effects of early-life stress. Nat. Neurosci. 12, 1559-U1108 (2009).

58. Veenema, A. H., Bredewold, R. \& Neumann, I. D. Opposite effects of maternal separation on intermale and maternal aggression in C57BL/6 mice: link to hypothalamic vasopressin and oxytocin immunoreactivity. Psychoneuroendocrinology 32, 437-450 (2007).

59. Donadon, M. F., Martin-Santos, R. \& Osorio, F. D. The associations between oxytocin and trauma in humans: a systematic review. Front. Pharmacol. 9, 16 (2018).

60. Brydges, N. M., Wood, E. R., Holmes, M. C. \& Hall, J. Prepubertal stress and hippocampal function: sex-specific effects. Hippocampus 24, 684-692 (2014).

61. Brydges, N. M. et al. Imaging conditioned fear circuitry using awake rodent fMRI. Plos ONE 8, e54197 (2013).

62. Brydges, N. M., Hall, L., Nicolson, R., Holmes, M. C. \& Hall, J. The effects of juvenile stress on anxiety, cognitive bias and decision making in adulthood: a rat model. Plos ONE 7, e48143 (2012).

63. Jacobson-Pick, S. \& Richter-Levin, G. Differential impact of juvenile stress and corticosterone in juvenility and in adulthood, in male and female rats. Behavioural Brain Res. 214, 268-276 (2010).

64. Hicks, C. et al. Body temperature and cardiac changes induced by peripherally administered oxytocin, vasopressin and the non-peptide oxytocin receptor agonist WAY 267,464: a biotelemetry study in rats. Br. J. Pharmacol. 171, 2868-2887 (2014).

65. Ramos, L. et al. Acute prosocial effects of oxytocin and vasopressin when given alone or in combination with 3,4-methylenedioxymethamphetamine in rats: involvement of the V1(A) receptor. Neuropsychopharmacology $\mathbf{3 8}$, 2249-2259 (2013).

66. Simola, N. \& Brudzynski, S. M. Rat 50-kHz ultrasonic vocalizations as a tool in studying neurochemical mechanisms that regulate positive emotional states. J. Neurosci. Methods 310, 33-44 (2018).

67. Leichsenring, F., Leibing, E., Kruse, J., New, A. S. \& Leweke, F. Borderline personality disorder. Lancet 377, 74-84 (2011).
68. Ekman, P. \& Friesen, W. Pictures of Facial Affect. (Consulting Psychologists Press, Palo Alto, CA, 1976).

69. Unoka, Z., Fogd, D., Fuzy, M. \& Csukly, G. Misreading the facial signs: specific impairments and error patterns in recognition of facial emotions with negative valence in borderline personality disorder. Psychiatry Res. 189, 419-425 (2011).

70. Wagner, A. W. \& Linehan, M. M. Facial expression recognition ability among women with borderline personality disorder: implications for emotion regulation? J. Personal. Disord. 13, 329-344 (1999).

71. Nicol, K., Pope, M. \& Hall, J. Facial emotion recognition in borderline personality: an association, with childhood experience. Psychiatry Res. $\mathbf{2 1 8}$ 256-258 (2014).

72. Christ-Crain, M. \& Fenske, W. Copeptin in the diagnosis of vasopressindependent disorders of fluid homeostasis. Nat. Rev. Endocrinol. 12, 168-176 (2016).

73. Balanescu, S. et al. Correlation of plasma copeptin and vasopressin concentrations in hypo-, iso-, and hyperosmolar states. J. Clin. Endocrinol. Metab. 96, 1046-1052 (2011).

74. Rotondo, F. et al. Arginine vasopressin (AVP): a review of its historical perspectives, current research and multifunctional role in the hypothalamohypophysial system. Pituitary 19, 345-355 (2016).

75. Aguilera, G. \& Rabadan-Diehl, C. Vasopressinergic regulation of the hypothalamic-pituitary-adrenal axis: implications for stress adaptation. Regulatory Pept. 96, 23-29 (2000).

76. Caldwell, H. K., Lee, H. J., Macbeth, A. H. \& Young, W. S. Vasopressin: behavioral roles of an "original" neuropeptide. Prog. Neurobiol. 84, 1-24 (2008).

77. Jasnic, N. et al. Distinct vasopressin content in the hypothalamic supraoptic and paraventricular nucleus of rats exposed to low and high ambient temperature. J. Therm. Biol. 52, 1-7 (2015).

78. Veenema, A. H., Blume, A., Niederle, D., Buwalda, B. \& Neumann, I. D. Effects of early life stress on adult male aggression and hypothalamic vasopressin and serotonin. Eur. J. Neurosci. 24, 1711-1720 (2006).

79. Perkeybile, A. M. \& Bales, K. L. Early rearing experience is related to altered aggression and vasopressin production following chronic social isolation in the prairie vole. Behav. Brain Res. 283, 37-46 (2015).

80. Perkeybile, A. M. \& Bales, K. L. Early rearing experience is associated with vasopressin immunoreactivity but not reactivity to an acute non-social stressor inthe prairie vole. Physiol. Behav. 147, 149-156 (2015).

81. Lee, P. R., Brady, D. L., Shapihro, R. A., Dorsa, D. M. \& Koenig, J. I. Prenatal stress generates deficits in rat social behavior: reversal by oxytocin. Brain Res. 1156, 152-167 (2007).

82. Oreland, S., Gustafsson-Ericson, L. \& Nylander, I. Short- and long-term consequences of different early environmental conditions on central immunoreactive oxytocin and arginine vasopressin levels in male rats. Neuropeptides 44, 391-398 (2010).

83. Avinun, R. et al. AVPR1A variant associated with preschoolers' lower altruistic behavior. Plos ONE 6, e25274 (2011).

84. Heim, C. et al. Lower CSF oxytocin concentrations in women with a history of childhood abuse. Mol. Psychiatry 14, 954-958 (2009).

85. Opacka-Juffry, J. \& Mohiyeddini, C. Experience of stress in childhood negatively correlates with plasma oxytocin concentration in adult men. Stress $\mathbf{1 5}$, $1-10$ (2012).

86. Mizuki, R. \& Fujiwara, T. Association of oxytocin level and less severe forms of childhood maltreatment history among healthy Japanese adults involved with child care. Front. Behav. Neurosci. 9, 138 (2015).

87. Tzanoulinou, S., Riccio, O., de Boer, M. W. \& Sandi, C. Peripubertal stressinduced behavioral changes are associated with altered expression of genes involved in excitation and inhibition in the amygdala. Transl. Psychiatry 4, e410 (2014).

88. Bielsky, I. F., Hu, S. B., Szegda, K. L., Westphal, H. \& Young, L. J. Profound impairment in social recognition and reduction in anxiety-like behavior in vasopressin V1a receptor knockout mice. Neuropsychopharmacology 29, 483-493 (2004).

89. Bielsky, I. F., Hu, S. B., Ren, X. H., Terwilliger, E. F. \& Young, L. J. The V1a vasopressin receptor is necessary and sufficient for normal social recognition: a gene replacement study. Neuron 47, 503-513 (2005).

90. Tobin, V. A. et al. An intrinsic vasopressin system in the olfactory bulb is involved in social recognition. Nature 464, 413-U110 (2010).

91. Dluzen, D. E., Muraoka, S. \& Landgraf, R. Olfactory bulb norepinephrine depletion abolishes vasopressin and oxytocin preservation of social recognition responses in rats. Neurosci. Lett. 254, 161-164 (1998). 
92. Gutzler, S. J., Karom, M., Erwin, W. D. \& Albers, H. E. Arginine-vasopressin and the regulation of aggression in female Syrian hamsters (Mesocricetus auratus). Eur. J. Neurosci. 31, 1655-1663 (2010).

93. Bester-Meredith, J. K. \& Marler, C. A. Vasopressin and aggression in crossfostered California mice (Peromyscus californicus) and white-footed mice (Peromyscus leucopus). Hormones Behav. 40, 51-64 (2001).

94. Delville, W., Mansour, K. M. \& Ferris, C. F. Testosterone facilitates aggression by modulating vasopressin receptors in the hypothalamus. Physiol. Behav. 60 25-29 (1996).

95. Knafo, A. et al. Individual differences in allocation of funds in the dictator game associated with length of the arginine vasopressin 1a receptor RS3 promoter region and correlation between RS3 length and hippocampal mRNA. Genes Brain Behav. 7, 266-275 (2008).

96. Uzefovsky, F. et al. Oxytocin receptor and vasopressin receptor 1a genes are respectively associated with emotional and cognitive empathy. Hormones Behav. 67, 60-65 (2015).

97. Cataldo, I., Azhari, A. \& Esposito, G. A review of oxytocin and argininevasopressin receptors and their modulation of autism spectrum disorder. Front. Mol. Neurosci. 11, 27 (2018).
98. Kim, S. J. et al. Transmission disequilibrium testing of arginine vasopressin receptor 1A (AVPR1A) polymorphisms in autism. Mol. Psychiatry 7, 503-507 (2002)

99. Guastella, A. J., Kenyon, A. R., Alvares, G. A., Carson, D. S. \& Hickie, I. B. Intranasal arginine vasopressin enhances the encoding of happy and angry faces in humans. Biol. Psychiatry 67, 1220-1222 (2010).

100. Thompson, R., Gupta, S., Miller, K., Mills, S. \& Orr, S. The effects of vasopressin on human facial responses related to social communication. Psychoneuroendocrinology 29, 35-48 (2004)

101. Vadas, L. et al. Sex-specific effect of intranasal vasopressin, but not oxytocin on emotional recognition and perception in schizophrenia patients. Eur. Psychiatry 41, S387-S388 (2017)

102. Dumais, K. M. \& Veenema, A. H. Vasopressin and oxytocin receptor systems in the brain: sex differences and sex-specific regulation of social behavior. Front. Neuroendocrinol. 40, 1-23 (2016).

103. Brydges, N. M. et al. Sex specific effects of pre-pubertal stress on hippocampal neurogenesis and behaviour. Transl. Psychiatry 8, 271 (2018).

104. Brydges N. M., Best C., Thomas K. L. Female HPA axis displays heightened sensitivity to pre-pubertal stress. Stress. https://doi.org/10.1080/10253890.2019. 